\title{
Right atrial pathology in arrhythmogenic right ventricular dysplasia
}

\author{
Guoliang Li ${ }^{1,2}$, Guy H. Fontaine ${ }^{2}$, Shuanliang Fan ${ }^{3}$, Yang Yan $^{4}$, \\ Peter K. Bode ${ }^{5}$, Firat Duru ${ }^{6}$, Robert Frank ${ }^{2}$, Ardan M. Saguner ${ }^{6}$ \\ ${ }^{1}$ Department of Cardiovascular Medicine, First Affiliated Hospital of Xi'an Jiaotong University, Xi'an, China \\ ${ }^{2}$ Cardiology Institute, Rhythmology Unit, Hôpital Universitaire La Pitié-Salpêtrière, Paris, France \\ ${ }^{3}$ Forensic Medical College of Xi'an Jiaotong University, Xi'an, China \\ ${ }^{4}$ Department of Cardiovascular Surgery, First Affiliated Hospital of Xi'an Jiaotong University, Xi'an, China \\ ${ }^{5}$ Institute of Pathology, University Hospital Zurich, Zurich, Switzerland \\ ${ }^{6}$ Department of Cardiology, University Heart Center, Zurich, Switzerland
}

\begin{abstract}
Background: Atrial fibrillation $(A F)$ is the most common atrial arrhythmia in arrhythmogenic right ventricular cardiomyopathy/dysplasia (ARVD). Considering the histologic changes known in the right ventricular (RV) in ARVD, the aim of the present study was to examine right atrial (RA) pathology in patients with ARVD.

Methods: Histology of $R A$ and $R V$ was assessed from autopsy material in 3 patients with ARVD without persistent atrial arrhythmia. RA histology in 3 patients with permanent AF without ARVD and 5 patients without cardiovascular disease was also studied. Staining with hematoxylin phloxine saffron was performed for the ARVD patients to identify fibrosis, and hematoxylin-eosin for identification of lymphocytes. Masson's trichrome staining was performed for control groups taken from a collection of standard glass slides.
\end{abstract}

Results: In all $3 A R V D$ cases, $R A$ anomalies were observed that revealed a reduction of cardiomyocytes, the presence of adipocytes, some of them inside the mediomural atrial layer and interstitial fibrosis. In $2 A R V D$ cases, interstitial fibrosis was also associated with a focus of replacement fibrosis, which was also observed in patients with permanent $A F$ without $A R V D$. The histologic specimen of the RA and $R V$ from the control group without cardiovascular disease did not display any evidence of fat or fibrosis with a preserved cardiomyocyte architecture.

Conclusions: A similar histopathological substrate, as can be observed in the $R V$ of patients with $A R V D$ can also be seen in the RA of these patients. This may explain the high prevalence of atrial arrhythmias, particularly AF, in patients with ARVD. (Cardiol J 2019; 26, 6: 736-743)

Key words: arrhythmogenic right ventricular dysplasia, atrial arrhythmias, pathological substrate, atrium

\section{Introduction}

Arrhythmogenic right ventricular cardiomyopathy/dysplasia (ARVD), first recognized in 1977, is an inherited cardiomyopathy mostly due to mutations in both desmosomal and non-desmosomal genes [1]. ARVD is considered as one of the lead- ing causes of sudden cardiac death in the young and among athletes [2, 3]. In patients with ARVD, a right ventricle (RV) covered by fat has been an impressive pattern observed after opening the pericardium before antiarrhythmic surgery (Fig. 1A). Histopathology of the RV in ARVD is studied at low magnification in order to display the full thickness

Address for correspondence: PD Dr. med. Ardan M. Saguner, Department of Cardiology, University Heart Center Zurich, Rämistrasse 100, 8091 Zurich, Switzerland, tel: +41-44-255-3422, fax: +41-44-255-4401, e-mail: Ardan.Saguner@usz.ch 


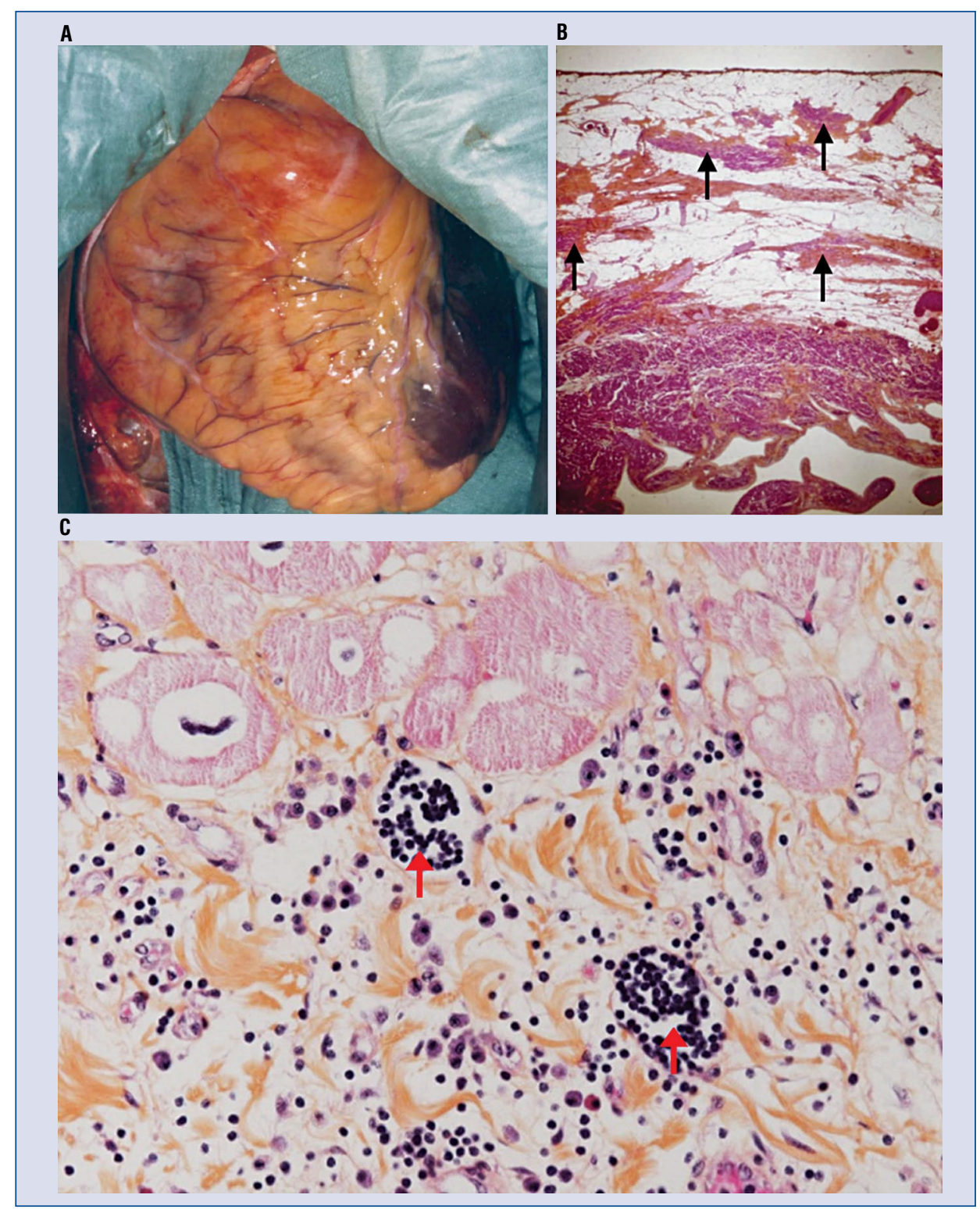

Figure 1. A. Surgical view of a typical right ventricular dysplasia (ARVD) beating heart. The right ventricle (RV) is dilated and totally covered by fat with extension over the left ventricle (LV) apex (dark red). Two aneurysms are visible on the infundibulum and the sub tricuspid area; B. Full thickness of the RV in a typical ARVD patient. Note the predominance of adipocytes in subepicardial layers and the presence of strands of cardiomyocytes (solid arrows) bordered by fibrosis within the fat, which is a marker of ARVD (HPS, 20×); C. Two foci (red solid arrows) of lymphocytes in zones of interstitial fibrosis in the RV. Also note the presence of eosinophils, monocytes and polymorphonuclears. This histologic specimen was obtained from a patient with ARVD, who was referred for heart transplantation due to a rapid deterioration of global cardiac function (HPS, 400×) (image was provided by Guy H. Fontaine).

of the free wall (Fig. 1B). This shows a particular topographic pattern in which a reduced amount of myocardium and a presence of fat and fibrosis are predominant features on epicardial layers. However, inside this fibro-fatty tissue, it is possible to observe strands of cardiomyocytes, which appear as a pathognomonic histological feature of this disease (Fig. 1B). It is also obvious that the preserved RV myocardium remains mostly apparent in the subendocardial layers frequently dissociated by excessive interstitial fibrosis (Fig. 1B). This can explain why trouble in conduction is mostly observed on epicardial layers, leading to identification of the Epsilon wave of postexcitation. However, fibrosis in the RV is twofold: 1) Interstitial fibrosis made of small layers of 
fibrous tissue bordering or embedded cardiomyocytes, and 2) Replacement fibrosis made of large amounts of hyaline fibrosis sometimes interspersed by a variable number of lymphocytes. These lymphocytes were later interpreted as a hallmark of superimposed myocarditis. This can be explained by the increased susceptibility of this abnormal myocardium in attracting inflammatory phenomena such as cardiotropic viruses, which can be a trigger of arrhythmias [4-6].

After the group around Guy Fontaine in Paris, which was the first to demonstrate a high prevalence of supraventricular arrhythmias in ARVD patients [7], this concept was further confirmed on a larger series of patients $[8,9]$. Of all atrial arrhythmias in ARVD, atrial fibrillation (AF) is the most common. A recent study of boxer dogs with histopathologically confirmed ARVD identified fibro-fatty infiltration and histopathologically altered intercalated disc and gap junction proteins in atrial myocardium, representing a substrate for atrial arrhythmias associated with ARVD [10]. This supports atrial involvement as part of this disorder. Therefore, this study examines the right atrial (RA) pathology of humans with ARVD in comparison to patients with persistent $\mathrm{AF}$ without ARVD and controls without cardiovascular disease (CVD), and considers typical histologic changes of the RV known in ARVD.

\section{Methods}

Right atrial histology was assessed in autopsy material from 3 patients with ARVD with atrial arrhythmias, but without a history of persistent AF. This was done in order to minimize the effects of atrial arrhythmia itself on atrial remodeling ("AF begets AF"). The diagnosis of ARVD was confirmed by clinical presentation and characteristic pathological and histopathological changes of the RV at autopsy. A regular technique of tissue processing for glass slides for light microscopic examination, with numeric image processing was performed. Staining with hematoxylin phloxine saffron (HPS) was performed for tissue obtained from the patients with ARVD to have a better identification of fibrosis, and hematoxylin-eosin (HE) for better identification of lymphocytes. Masson's trichrome staining was performed for two control groups taken from a collection of standard glass slides. Examination of all slides was made at low $(20 \times)$ and high magnification $(400 \times)$ with special respect to adipocytes, interstitial or replacement fibrosis as well as high magnification for detection of clusters of lymphocytes isolated in myocardium or embed- ded in fibrosis, which represent a chronic-active form of myocarditis. The RA samples from patients with permanent $A F$ without ARVD were obtained from the First Affiliated Hospital of Xi'an Jiaotong University, Xi'an, China. RA autopsy samples from individuals without CVD were obtained from the Forensic Medical College of Xi'an Jiaotong, University Health Science Center, China. This was approved by the ethical committee of Xi'an Jiaotong University Health Science Center, China (approval number: 2018007). Informed consent was obtained from all patients or their relatives. Autopsy samples of patients with ARVD from Hôpital de la Salpêtrière, Paris, France, were taken before 1975. Three patients with ARVD did not refuse research associated with their disease, and at that time there was no ethics committee, but Chief of the Department of Cardiology, Hôpital de la Salpêtrière, Paris, France approved the investigation to learn about the etiology of arrhythmias in these patients.

\section{Results}

\section{ARVD group}

To improve clarity, magnification and staining are indicated in each picture. The reference histologic document of a typical RV free wall, changes in a characteristic patient with ARVD (case 1) is presented in Figure 1B. Note the value of low magnification to display the full thickness of the RV free wall (Fig. 1B) and the value of high magnification in assessing lymphocytes (Fig. 1C).

In summary, reference histological material of the RV shows:

- reduction of cardiomyocytes;

- continuous adipose tissue on the epicardium;

- strands of cardiomyocytes within adipose tissue in the subepicardial layers;

- strands of fibrous tissue all over the free wall, being more severe on endocardial layers;

- paucity or absence of cardiomyocytes in medio-mural layers.

\section{Case 1}

The patient was a 28 -year-old Caucasian male, $54 \mathrm{~kg}$, born in the Veneto region, Italy. His familial history was not available. Transthoracic echocardiography had shown a left ventricular ejection fraction (LVEF) of $25 \%$ and RV fractional area change of $20 \%$ after 10 years follow-up for paroxyxmal ventricular tachycardia (VT). The patient experienced almost incessant VT of four different morphologies and then was referred to the present group in Paris for antiarrhythmic surgery. 
Ablation by 17 shock of 100 joules delivered by direct current was performed to prevent relapses of VT due to a very high risk for open heart surgery. However, this patient died one week later of a longstanding pulmonary infection prior to the ablation procedure. Autopsy was performed the same day. Gross pathology studied before preservation in formalin confirmed the diagnosis of ARVD, the whole RV was covered by fat. A major increase of trabeculations was observed after opening the $\mathrm{RV}$ free wall, which was paper-thin and showed a significant reduction of myocardial tissue (Fig. 2A). The RA histological sample from this patient is also impressive by the major evidence of interstitial fibrosis and small islands of adipocytes embedded or bordered by residual cardiomyocytes, which were considerably reduced in amount (Fig. 2B). Also note an increased thickness of the media layer of the distal coronary vessel, which is well in line with the concept of the presence of cardiac microvascular disease in ARVD [11] and may be responsible for one of the mechanisms for chest pain in ARVD patients [2, 12].

\section{Case 2}

The patient was a 48-year-old male of African descent, born in La Martinique, $90 \mathrm{~kg}$, LVEF $25 \%$. He had a history of paroxysmal AF and was referred for VT ablation after several episodes of tolerated sustained VT. However, this patient died due to cardiac tamponade after insertion of the RV endocardial catheters before ablation. The pathology of the RV was typical in ARVD. The histological study of the RA (Fig. 2C) showed evidence of interstitial fibrosis bordering or embedding a reduced number of cardiomyocytes, but was less severe than in the previous case. Presence of some clusters of adipocytes inside the myocardium was also noted. A focus of replacement fibrosis on the epicardial layer with strands of fibrosis towards the endocardial layer was present. This pattern suggests sequelae of healed - epicardial towards endocardial - myocarditis. An absence of lymphocytes is noticeable.

\section{Case 3}

This patient was a Caucasian woman in the her late 30 s, who had died in hospital of intractable VT leading to an autopsy and subsequent typical histologic diagnosis of ARVD. Histology of the RA showed zones of major fibrosis and others with excessive fat containing residual strands of cardiomyocytes, which were considerably reduced in amount (Fig. 3A, B). Histology of the RV revealed typical characteristics of ARVD (Fig. 3C).

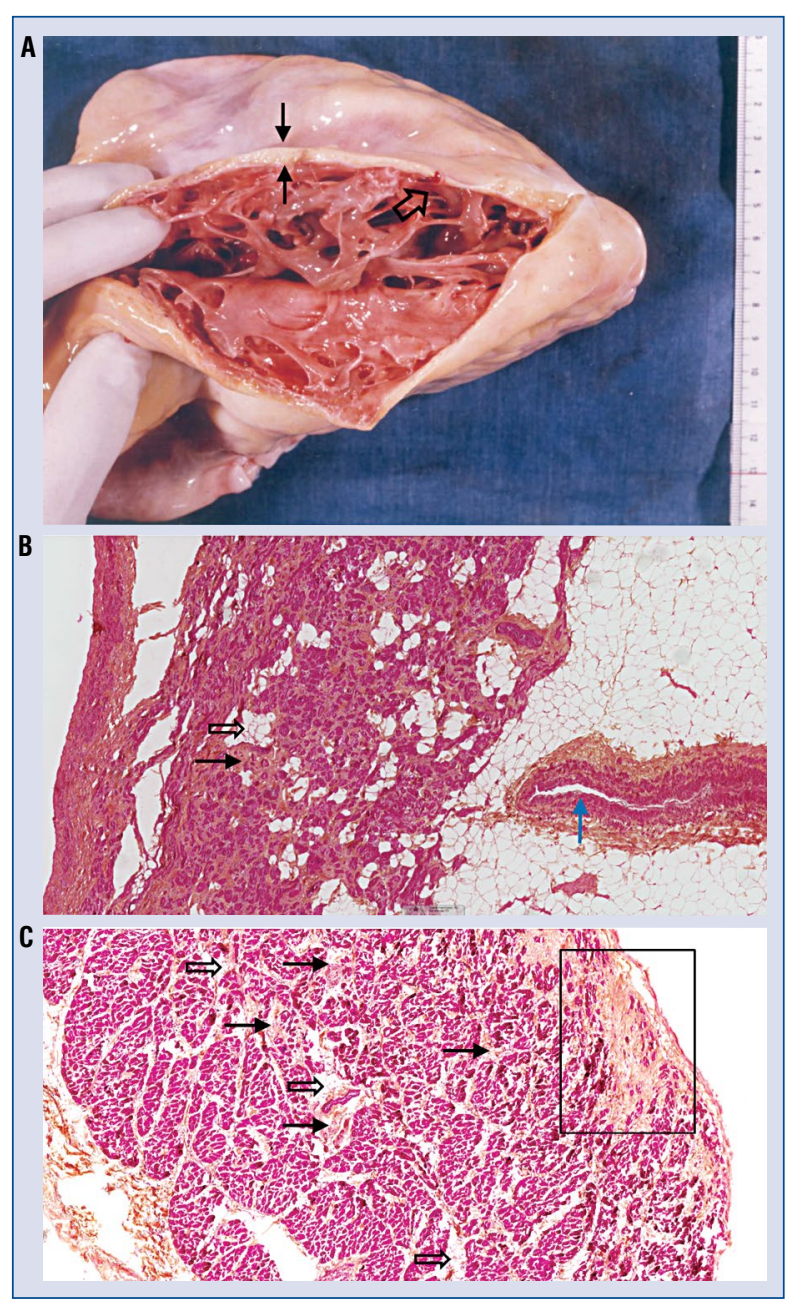

Figure 2. A. The heart is cut from apex to base along the acute margin of the right ventricle (RV) free wall. Note that all the epicardium is covered by fat (black solid arrows). Myocardium remains only on a thin endocardial layer. Also note an increased number of trabeculations (black open arrows). The heart was obtained immediately after the patient's death, before preservation in formalin to maintain the native color of the tissue. Ventricular tachycardia was ablated after 17 shocks of 100 joules delivered in the RV, however, this document shows that no scar was visible on the endocardium (Courtesy of Pr Piccolo, Venice, Italy); B. Sample of the right atrial free wall of the same right ventricular dysplasia (ARVD) patient presented on panel $A$. This sample is impressive showing major evidence of interstitial fibrosis (black solid arrows) and clusters of adipocytes (black open arrows) bordering cardiomyocytes. Also note an increased thickness of the media layer of a distal coronary vessel (blue solid arrows, micro-vascular disease concept, see text); C. Evidence of interstitial fibrosis (black solid arrows) bordering or embedding cardiomyocytes, which is less severe than in the previous case. The presence of some clusters of adipocytes (black open arrows) inside myocardium is visible. The presence of fibrosis (black square) on the epicardial layer with strands of fibrosis towards endocardial layers was observed. This pattern suggests sequel of healed epicardial towards endocardial myocarditis. An absence of lymphocytes is noticeable (image was provided by Guy H. Fontaine). 


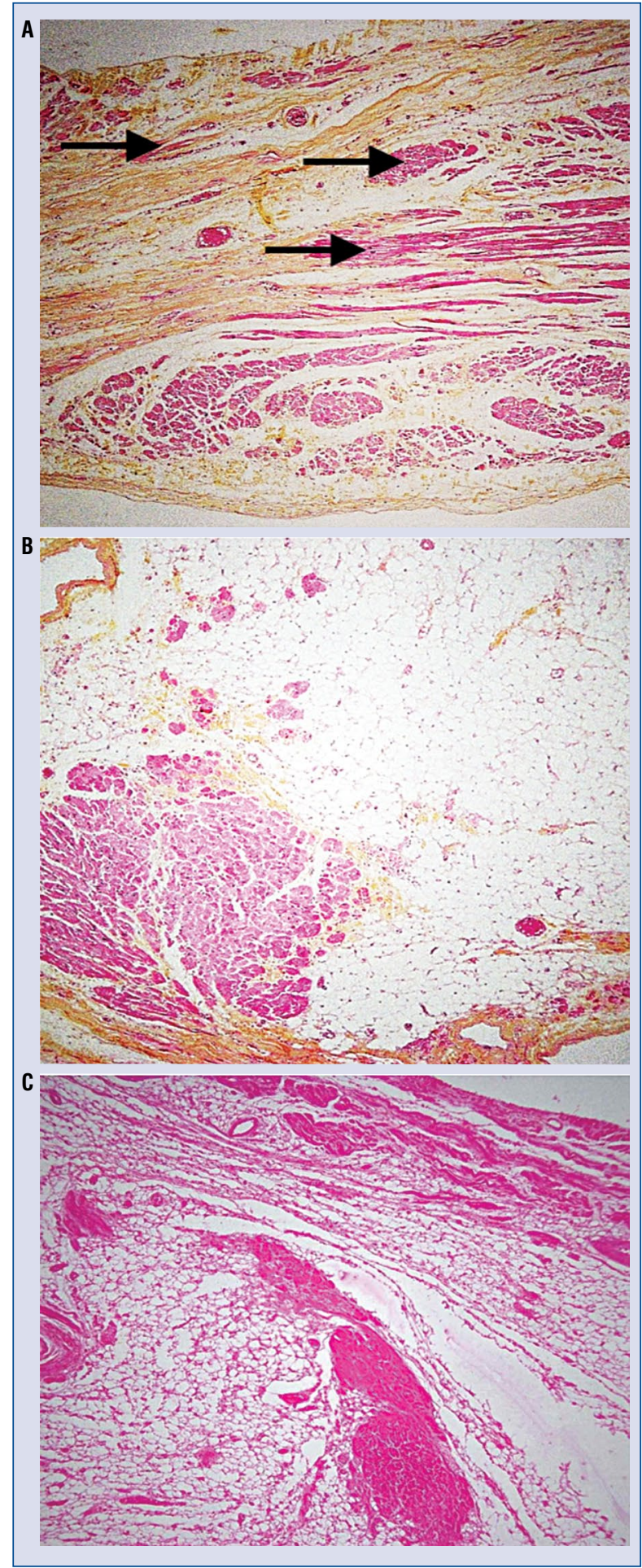

Figure 3. Third patient showing right ventricular dysplasia (ARVD). A. Major dissociation of strands of cardiomyocytes (black solid arrows) by fibrosis in the right atrial free wall; B. Presence of an excessive amount of epicardial adipocytes occupied by strands of cardiomyocytes in right atrial tissue; C. Histology of the right ventricle in this third patient shows a major predominance of adipocytes and fibrosis, a typical characteristic of ARVD (image was provided by Guy H. Fontaine).

\section{Control groups}

Three patients with permanent AF without ARVD were chosen as a positive control group (Fig. 4A). They were $>50$ years; two were males. Their RA histology (Fig. 4B) shows a major predominance of adipocytes and fibrosis. Five individuals without known CVD, had died of a non-cardiac cause served as negative controls, three were males. They showed a pattern of a homogenous myocardial structure exempt of fat, fibrosis or inflammation (Fig. 4C, 4D).

\section{Discussion}

In this autopsy study of RA tissue from patients with ARVD without a history of persistent atrial arrhythmias demonstrated similar histopathological changes when compared to typical RV changes of fibro-fatty infiltration known in ARVD. According to available research, this is the first human study investigating RA histology of patients with ARVD as compared to patients with permanent AF without ARVD and in patients without any CVD. The atrial histopathological changes observed in the ARVD cohort were absent in controls without CVD who had died of a non-cardiac cause, but a similar pattern of RA fibro-fatty infiltration was observed in patients with permanent AF without ARVD.

The pathologic changes observed in the RA are similar to those known in the right (or possibly left ventricle) ARVD. The first case of this series displays a typical picture of what is considered "atrial dysplasia". This term was first reported by the present group in a patient with myotonic dystrophy where the dysplastic phenomenon was localised in a focal zone [13]. The second case shows evidence of diffuse interstitial fibrosis with small clusters of adipocytes inside the atrial myocardium. Another very important anomaly was the patchy distribution of zones of epicardial hyaline fibrosis in agreement with a pattern of healed myocarditis, probably superimposed on the genetically produced dysplastic phenomenon. This pattern suggests sequelae of healed epicardial towards endocardial myocarditis. An increased thickness of the media layer in a distal coronary vessel is in line with the presence of a microvascular disease in ARVD [11], which can explain atypical chest pain in some of these patients [2, 14].

Fibrofatty atrial infiltration has already been described in patients with persistent AF [15]. In the present study, the atrial myocardium in patients with ARVD and those with permanent AF was not made of compact myocardium such as the 


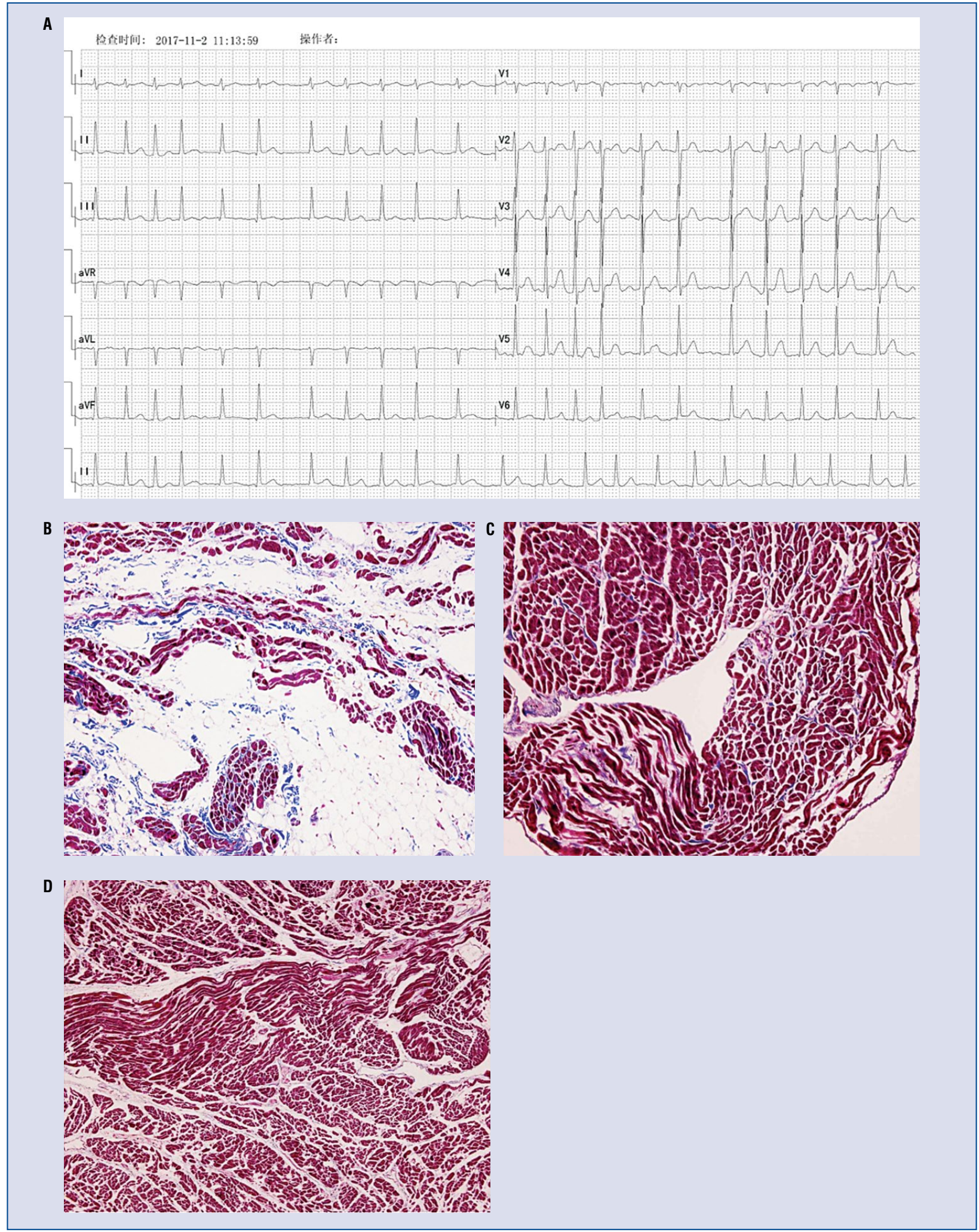

Figure 4. A 46-year-old female patient was diagnosed with permanent atrial fibrillation, without right ventricular cardiomyopathy/dysplasia (ARVD) (A). Histology of the right atrium (B) in this patient shows a major predominance of adipocytes and fibrosis, similar to that observed in patients with ARVD (Masson's trichrome, 10×). Right atrial (C) and right ventricular (D) histology of a 21-year-old male without known cardiovascular disease, who died of a non-cardiac cause, shows a homogenous myocardial structure exempt of fat, fibrosis or inflammation (Masson's trichrome, 20×) (image was provided by Guy H. Fontaine). 
homogenous structure observed in the normal left ventricle, but showed a spectrum of anomalies known in the RV of patients with ARVD. Of importance, atrial dysplasia in patients with ARVD was present in the absence of a history of persistent atrial arrhythmias. Therefore, it can be hypothesized that atrial dysplasia is not directly related to the presence of atrial arrhythmias in these patients, but should follow a different pathophysiologic mechanism. As expected, these anomalies were absent in patients without CVD.

Concepts herein are clear and simple because they are based on obvious structural heart disease. However, this understanding requires basic knowledge in the field of histology of the myocardium under normal and pathologic conditions. This pathological substrate most likely creates the basis for AF, similar to ventricular arrhythmias well known in patients with ARVD $[2,6,16]$. Yet, the specific mechanisms leading to atrial dysplasia in ARVD remain unclear and require further investigation in future studies. A primary disease of the atria, as suggested in studies of ARVD boxer dogs is possible [10]. However, it is also possible that right and left ventricle dysfunction and adverse ventricular remodeling secondarily leads to increased loading conditions in the atria, which produces atrial dysplasia, similar to patients with persistent AF without ARVD $[9,17]$.

In short, histologic anomalies in the human ventricular myocardium may lead to ventricular arrhythmias, and in more severe cases, to ventricular fibrillation and sudden death. The same situation, which also seems to be common in the atria, can lead to atrial arrhythmias including AF.

\section{Limitations of the study}

This preliminary report was performed with small series of patients with ARVD since it is challenging to obtain atrial tissue for histologic examination from these patients. Moreover, genotyping was not available when these samples were collected. However, the clinical investigation, gross pathology and histopathology were well in line with a diagnosis of ARVD in these 3 patients. From the present descriptive study, it cannot be inferred as to whether the structural changes in the RA of patients with ARVD are secondary due to RV failure, or are primarily due to genetic mutations or epigenetic factors that affect the intercalated disc of atrial tissue. Atrial tissue was only available from the RA. Therefore, histological findings from the left atrium cannot report on, which is important for triggering and maintaining AF in patients without ARVD.

\section{Conclusions}

This histologic study of RA tissue in 3 patients with ARVD without a history of persistent atrial arrhythmias demonstrates structural changes similar to those of the RV known in ARVD. The present findings help to explain the underlying substrate and high prevalence of $\mathrm{AF}$ in this cardiomyopathy.

\section{Acknowledgements}

Dr. Guoliang Li has worked with Guy H. Fontaine's group in Paris since 2015 and was supported by the Clinical Research Award of the First Affiliated Hospital of Xi'an Jiaotong University, China (No. XJTU1AF-CRF-2018-015; No. XJTU1AFCRF2015007). The Zurich ARVC Program is supported by grants from the Bertha and Georg Schwyzer-Winiker Foundation, Baugarten Foundation, both in Zurich, Switzerland and the Swiss National Science Foundation.

We dedicate this work to our great mentor and teacher Guy H. Fontaine who is deeply missed.

\section{Conflict of interest: None declared}

\section{References}

1. Fontaine G, Guiraudon G, Frank R, et al. Stimulation studies and eicardial maing in ventricular tachycardia: study of mechanisms and selection for surgery. In: Kulbertus H, editor.: Lancaster. MTP Pub. 1977: 334-350.

2. Marcus FI, Fontaine GH, Guiraudon G, et al. Right ventricular dysplasia: a report of 24 adult cases. Circulation. 1982; 65(2): 384-398, indexed in Pubmed: 7053899.

3. Calkins H, Corrado D, Marcus F. Risk stratification in arrhythmogenic right ventricular cardiomyopathy. Circulation. 2017; 136(21): 2068-2082, doi: 10.1161/CIRCULATIONAHA.117.030792, indexed in Pubmed: 29158215.

4. Lopez-Ayala JM, Pastor-Quirante F, Gonzalez-Carrillo J, et al. Genetics of myocarditis in arrhythmogenic right ventricular dysplasia. Heart Rhythm. 2015; 12(4): 766-773, doi: 10.1016/j. hrthm.2015.01.001, indexed in Pubmed: 25616123.

5. Bowles NE, Ni J, Marcus F, et al. The detection of cardiotropic viruses in the myocardium of patients with arrhythmogenic right ventricular dysplasia/cardiomyopathy. J Am Coll Cardiol. 2002; 39(5): 892-895, indexed in Pubmed: 11869858.

6. Saguner AM, Roland F, Li GL, et al. 1933Superimposed myocarditis leading to heart transplantation in a young patient with arrhythmogenic right ventricular dysplasia. Eur Heart J. 2017; 38(Suppl. 1), doi: 10.1093/eurheartj/ehx495.1933.

7. Tonet JL, Castro-Miranda R, Iwa T, et al. Frequency of supraventricular tachyarrhythmias in arrhythmogenic right ventricular dysplasia. Am J Cardiol. 1991; 67(13): 1153, indexed in Pubmed: 2024612.

8. Saguner AM, Ganahl S, Kraus A, et al. Clinical role of atrial arrhythmias in patients with arrhythmogenic right ventricular dysplasia. Circ J. 2014; 78(12): 2854-2861, indexed in Pubmed: 25327952.

9. Wu L, Guo J, Zheng L, et al. Atrial remodeling and atrial tachyarrhythmias in arrhythmogenic right ventricular cardiomyopa- 
thy. Am J Cardiol. 2016; 118(5): 750-753, doi: 10.1016/j.amjcard.2016.06.003, indexed in Pubmed: 27378141.

10. Vila J, Pariaut R, Moïse NS, et al. Structural and molecular pathology of the atrium in boxer arrhythmogenic right ventricular cardiomyopathy. J Vet Cardiol. 2017; 19(1): 57-67, doi: 10.1016/j. jvc.2016.09.001, indexed in Pubmed: 27769725.

11. Fontaine G, Fornes P, Hebert JL. Ventricular tachycardia in arrhythmogenic right ventricular cardiomyoathies. In: Zies D, Jalife J, editors. Cardiac Electrohysiology: From Cell to Bedside (4th edition). Saunders, Philadelphia 2004; 588-600.

12. Paul M, Rahbar K, Gerss J, et al. Microvascular dysfunction in nonfailing arrhythmogenic right ventricular cardiomyopathy. Eur J Nucl Med Mol Imaging. 2012; 39(3): 416-420, doi: 10.1007/ /s00259-011-1985-8, indexed in Pubmed: 22113617.

13. Bonny A, Lellouche N, Ditah I, et al. C-reactive protein in arrhythmogenic right ventricular dysplasia/cardiomyopathy and relationship with ventricular tachycardia. Cardiol Res Pract. 2010; 2010, doi: 10.4061/2010/919783, indexed in Pubmed: 20885777.
14. Spoladore R, Fisicaro A, Faccini A, et al. Coronary microvascular dysfunction in primary cardiomyopathies. Heart. 2014; 100(10): 806-813, doi:10.1136/heartjnl-2013-304291, indexed in Pubmed: 23904360.

15. Haemers P, Hamdi H, Guedj K, et al. Atrial fibrillation is associated with the fibrotic remodelling of adipose tissue in the subepicardium of human and sheep atria. Eur Heart J. 2017; 38(1): 53-61, doi: 10.1093/eurheartj/ehv625, indexed in Pubmed: 26612579.

16. Saguner AM, Brunckhorst C, Duru F. Atrial arrhythmias in arrhythmogenic cardiomyopathy: at the beginning or at the end of the disease story? Reply. Circ J. 2015; 79(2): 447, doi: 10.1253/ circj.CJ-14-1234, indexed in Pubmed: 25482384.

17. Platonov PG, Christensen AH, Holmqvist F, et al. Abnormal atrial activation is common in patients with arrhythmogenic right ventricular cardiomyopathy. J Electrocardiol. 2011; 44(2): 237-241, doi: 10.1016/j.jelectrocard.2010.08.008, indexed in Pubmed: 21093870. 